\title{
Marcadores bioquímicos, fisiológicos y celulares asociados con lesión pulmonar aguda (LPA/SDRA)
}

\author{
Francisco Hernández Gómez-Crespo, *, Raúl Barrera-Rodríguez,* ${ }^{*}$ Jorge Chavarría-Garcés* \\ *Instituto Nacional de Enfermedades Respiratorias Ismael Cosío Villegas, Ciudad de México; *Facultad de Medicina, \\ Universidad Westhill. \\ Trabajo recibido: 21-I-2015; aceptado: 13-II-2015
}

\begin{abstract}
RESUMEN. El síndrome de dificultad respiratoria aguda (SDRA) es secundario a una lesión pulmonar o extrapulmonar aguda, que conduce al daño de la membrana alveolocapilar pulmonar con aumento de la permeabilidad. La descripción clínica del síndrome se ha abordado en varias revisiones; sin embargo, aún es difícil predecir el comportamiento clínico que seguirán algunos pacientes, ya que mientras unos se agravan rápidamente otros logran su mejoría. El conocimiento de los aspectos moleculares que regulan los procesos fisiológicos serán abordados en esta revisión, con la finalidad de identificar posibles marcadores genéticos de valor predictivo que permitan identificar a aquellos pacientes que podrían ser más susceptibles a desarrollar SDRA, o bien al grupo de pacientes con mayor probabilidad de tener una mejor respuesta a un tratamiento determinado, así como el pronóstico de los pacientes individuales.
\end{abstract}

Palabras clave: Lesión pulmonar aguda, síndrome de dificultad respiratoria aguda, biomarcadores, tratamiento.

ABSTRACT. ARDS is a clinical syndrome secondary to acute lung injury, which leads to damage of the alveolar pulmonary capillary membrane, with increased permeability. The clinical description of the syndrome has been discussed in several reviews; however, it's still difficult to predict the clinical behavior that patients will have, some of them decay rapidly while others quickly achieve their improvement. Knowledge of the molecular aspects that regulate physiological processes will be discussed in this review, in order to identify possible genetic markers, predictive value to identify patients who may be more susceptible to develop ARDS, or a patient group more likely to have a better response to a particular treatment and the prognosis of individual patients.

Key words: Acute lung injury, respiratory distress syndrome, biomarkers, treatment outcome.

Abreviaturas:

UCI: Unidades de Cuidados Intensivos.

LBA: Lavado bronquioalveolar.

LPA: Lesión pulmonar aguda.

SDRA: Síndrome de Dificultad Respiratoria Aguda.

\section{INTRODUCCIÓN}

El síndrome de dificultad respiratoria aguda (SDRA), es el término utilizado para designar varias formas agudas o subagudas de lesión pulmonar difusa que causan hipoxemia severa. Estas lesiones se asocian a una gran variedad de factores precipitantes y no dependen de ningún tipo de insuficiencia cardiocirculatoria concomitante, por lo que se manifiesta sin incremento de las presiones en los capilares pulmonares. EI SDRA es un síndrome clínico común y devastador asociado a la lesión pulmonar aguda (LPA) que se presenta como complicación por agresiones pulmonares tanto directas (i.e. neumonía) como indirectas (i.e., sepsis), y con frecuencia exige internación en las unidades de cuidados intensivos (UCI). ${ }^{1}$

Los factores que predicen la aparición o la gravedad del SDRA están pobremente definidos. Sin embargo, la incidencia relativamente baja de SDRA en el grupo relativamente grande de pacientes con riesgo, sugiere que los factores genéticos deben contribuir a la progresión del síndrome.

El propósito de esta revisión es describir el estado actual de las moléculas que tienen una participación importante en la predisposición o desarrollo del SDRA y su utilidad como biomarcadores pronósticos.

\section{EPIDEMIOLOGÍA}

Los estudios epidemiológicos de SDRA son difíciles de encontrar debido a dos factores. En primer lugar, los problemas con la fiabilidad de los diagnósticos y 
las inexactitudes de codificación administrativa hacen que los estudios de epidemiología basados en población sean costosos y difíciles, porque es necesario un control cuidadoso de calidad y validación prospectiva de las cohortes. En segundo lugar, dado que la LPA es una enfermedad grave y requiere gasometría arterial y radiografías de tórax para el diagnóstico, y a que los pacientes son atendidos en la $\mathrm{UCl}$, la epidemiología del síndrome está indisolublemente ligada a los servicios de salud.

No obstante, algunos estudios epidemiológicos muestran una variabilidad considerable en la incidencia de SDRA, p. ej., en Estados Unidos se registra una incidencia de 78.9 por cada 150,000 habitantes, mientras que en los países escandinavos es de 13.5, en Escocia de 16, en Australia 28, en Brasil (Vitoria) 64.2 con una mortalidad del 30 al $40 \%$ en esta última. ${ }^{2,3}$ Estos números hacen hincapié en la necesidad no sólo de obtener un método de diagnóstico precoz, sino también de poder identificar factores pronósticos de gravedad, y a partir de estos resultados tener la posibilidad de tomar mejores decisiones terapéuticas.

\section{DEFINICIÓN DEL SDRA}

La conferencia de Consenso Americano-Europea define la LPA y a su forma más grave, el SDRA como «un síndrome de inflamación y aumento de la permeabilidad que se asocia con una constelación de anomalías clínicas, radiológicas y fisiológicas que no pueden ser explicadas, pero pueden coexistir sin disfunción de la aurícula izquierda o hipertensión pulmonar». Debido a los problemas de fiabilidad y validez, la definición fue actualizada en el año 2012 por la Sociedad Europea de Medicina Intensiva. La nueva definición separa el SDRA en tres categorías basadas en la relación entre la presión parcial de oxígeno arterial $\left(\mathrm{PaO}_{2}\right)$ a la fracción de oxígeno inspirado $\left(\mathrm{FiO}_{2}\right)$. Las tres categorías son: leve $\left(200 \mathrm{mmHg}<\mathrm{PaO}_{2} / \mathrm{FiO}_{2} \leq 300 \mathrm{mmHg}\right.$ ); moderada (100 $\left.\mathrm{mmHg}<\mathrm{PaO}_{2} / \mathrm{FiO}_{2} \leq 200 \mathrm{mmHg}\right)$ y grave $\left(\mathrm{PaO}_{2} / \mathrm{FiO}_{2} \leq\right.$ $100 \mathrm{mmHg}){ }^{4}$ Esta nueva definición ha demostrado ser un mejor indicador para predecir la aparición de SDRA, como se ha demostrado con el análisis de área bajo la curva para la característica operativa del receptor (ROC) de $0.577 \%$ en comparación con la anterior definición, donde había un ROC de $0.536 \% .4,5$ Aunque ninguna de las dos definiciones resulta buena en predecir la mortalidad de estos pacientes.

\section{PATOLOGÍA}

La patología que conduce a LPA/SDRA no se comprende bien, sobre todo por la heterogeneidad de las condiciones provocadas por la misma. Los estudios de autopsia muestran que hay una serie de características patológicas pulmonares comunes, como el compromiso de la barrera alveolocapilar, lo que lleva a la formación del edema en el intersticio, así como en el espacio alveolar. $^{6}$

La patología del SDRA puede progresar a través de tres etapas superpuestas: a) fase inflamatoria o exudativa (1-7 días), b) proliferativa (7-21 días), c) fibrótica (> 21 días). La fase exudativa corresponde a la etapa inflamatoria aguda de SDRA y se caracteriza por la liberación de citocinas proinflamatorias, afluencia de neutrófilos. Uno de los sitios primarios afectados dentro de un proceso inflamatorio es la membrana alveolocapilar, que consta de un endotelio capilar, la membrana basal capilar, una membrana basal epitelial y un epitelio alveolar. Los neumocitos tipo I forman $90 \%$ de la capa epitelial alveolar, pero son muy susceptibles de ser dañados, mientras que los neumocitos tipo Il componen el otro $10 \%$ y son más resistentes, situación por demás muy importante dada su capacidad para producir surfactantes, transportar iones, proliferar y diferenciarse hacia neumocitos tipo I. La alteración del endotelio vascular lleva a la filtración de un líquido rico en proteínas dentro del intersticio circundante. Además, los daños causados a las uniones estrechas formados por los neumocitos tipo I en el tejido alveolar permiten al líquido proteínico permear e inundar el alvéolo. Colectivamente, los daños producidos a la capa alveolocapilar, llevan a la formación de edema pulmonar, característico de la fase temprana exudativa del SDRA.

Estos acontecimientos tempranos son seguidos por la fase proliferativa, que se desarrolla en la segunda y tercera semana (aunque puede iniciar simultáneamente con la fase exudativa y sobreponerse). Durante esta fase sigue el drenado del líquido de edema causado por la primera etapa y se caracteriza por la proliferación de los fibroblastos e hiperplasia de neumocitos tipo II que reemplazan a los tipos I; estos dos tipos de células están involucradas en la reparación de la membrana alveolocapilar. Para lograr una restauración, el tejido cicatrizal formado por los fibroblastos debe ser remodelado por los neumocitos tipo II, el edema debe de ser completamente drenado y las células y mediadores inflamatorios tienen que ser suprimidos. La excesiva deposición de colágeno por los fibroblastos, junto con el fracaso de la reparación de la arquitectura normal del alvéolo por las células tipo II, pueden dar lugar a la lesión pulmonar fibrótica permanente. Se cree que las células inflamatorias y las citoquinas juegan un papel en esta respuesta errónea. ${ }^{7}$ Los hallazgos histopatológicos están en relación con el tiempo de evolución del SDRA, de forma que hacia el día 35 después del 
inicio del cuadro, es imposible distinguir entre la fibrosis intraluminal y la intersticial. ${ }^{8}$

Los pacientes con SDRA suelen recuperar la función pulmonar de tres a cuatro semanas después de actuar el elemento lesivo en los pulmones, pero inician una fase fibrótica que requiere a veces de apoyo con ventilador mecánico, oxígeno suplementario o ambas medidas por largo tiempo. Con respecto a la imagen histológica, el edema alveolar y los exudados inflamatorios de fases anteriores se transforman en fibrosis pulmonar extensa de los conductos y del plano intersticial. La arquitectura de los acinos se altera extraordinariamente. Las consecuencias funcionales incluyen un mayor peligro de neumotórax, disminución de la distensibilidad pulmonar e incremento del espacio muerto de los pulmones. Aproximadamente dos tercios de pacientes que sobrevivieron a un episodio de SDRA tienen función pulmonar anormal un año después. Se han realizado estudios en pacientes sobrevivientes nueve meses después al SDRA y se ha observado fibrosis intersticial ligera, hiperplasia epitelial e incremento de macrófagos alveolares con linfocitos intersticiales. Los hallazgos de fibrosis en la biopsia pulmonar en cualquier fase del SDRA conllevan un incremento en la mortalidad. ${ }^{6-8}$

\section{MARCADORES MOLECULARES ASOCIADOS CON EL SDRA}

Un hecho que llama la atención es por qué algunos pacientes con SDRA por inflamación no controlada o sepsis se agravan rápidamente, mientras que otros logran su mejoría. Algunas respuestas a estas interrogantes han tratado de ser explicadas a través del conocimiento de los eventos celulares y moleculares que regulan, tanto el proceso inflamatorio como el daño y reparación de los tejidos. En esta intrincada red de posibilidades, los polimorfismos génicos (variaciones genéticas que se manifiestan alrededor del $1 \%$ de la población) parecen en ocasiones tener alguna relación con el comportamiento clínico de la enfermedad. Recién se han escrito varios estudios genéticos que muestran cómo el perfil de expresión génica está fuertemente asociado con el tipo específico de daño pulmonar y el pronóstico del mismo.

En el SDRA existen varios candidatos asociados con la susceptibilidad, curso clínico y pronóstico. Muchos de los genes y sus productos están relacionados con la inflamación, la respuesta inmune, la permeabilidad vascular, el tono vascular, los procesos de reparación, quimiotaxis, motilidad celular y coagulación. Desde el punto de vista del médico resulta importante saber si un marcador genético puede ser un buen indicador predictivo que permita identificar a aquellos pacientes que podrían ser más susceptibles a desarrollar SDRA, o bien al grupo de pacientes con mayor probabilidad de tener una mejor respuesta a un tratamiento determinado, así como el pronóstico de los pacientes individuales. Algunas de las respuestas a estas interrogantes han tratado de ser explicadas a través del conocimiento de los eventos celulares y moleculares que regulan, tanto el proceso inflamatorio como el daño y reparación de los tejidos.

Se han llevado a cabo muchos estudios para tratar de identificar indicadores útiles para ayudar en el diagnóstico y pronóstico. Hasta hoy, no existen estudios validados que permitan asignar un marcador pronóstico definitivo.

\section{MOLÉCULAS DE INFLAMACIÓN}

Es ampliamente aceptado que la formación de mediadores inflamatorios desempeña un papel importante en la fisiopatología del SDRA. Las moléculas que participan en este proceso son señaladas en la tabla 1. Es importante resaltar que, al presente, sólo unas pocas de ellas han sido validadas por estudios multicéntricos y no se ha podido encontrar ninguna que demuestre ser útil en la práctica clínica como biomarcador de susceptibilidad a SDRA.

\section{PROTEÍNAS RELACIONADAS A CAMBIOS EN LA PERMEABILIDAD Y TONO VASCULAR}

En el SDRA el intercambio de gases es afectado por el aumento en la dispersión de la ventilación alveolar y el gasto cardíaco debido a que ambas, tanto las funciones bronquiales como las vasculares se ven afectadas por factores relacionados con la enfermedad, tales como los efectos de los mediadores inflamatorios en las vías respiratorias y el tono del músculo liso vascular. Asimismo, las afecciones a la microcirculación pulmonar conducen la patogénesis del SDRA, porque el contenido de agua de los pulmones depende de la integridad de la barrera endotelial. Las moléculas asociadas con estos cambios se muestran en la tabla 2.

\section{MARCADORES RELACIONADOS CON LA TENSIÓN SUPERFICIAL, QUIMIOTAXIS Y MOTILIDAD CELULAR}

Las lesiones en el epitelio o endotelio alveolar dan como resultado la acumulación de edema rico en proteínas en sus espacios aéreos que inactivan el surfactante y aumentan la distancia de difusión para el intercambio de gases. El drenado del líquido desde 
Tabla 1. Marcadores relacionados al proceso inflamatorio en SDRA.

\begin{tabular}{lll}
\hline Molécula & \multicolumn{1}{c}{ Fuente } & \multicolumn{1}{c}{ Función } \\
\hline TNF- $\alpha$ & $\begin{array}{l}\text { Macrófagos células epiteliales, } \\
\text { endoteliales y fibroblastos }\end{array}$ & $\begin{array}{l}\text { Participa en la formación de } \\
\text { edema inhibiendo su reabsorción }\end{array}$ \\
& $\begin{array}{l}\text { Un polimorfismo en la región } \\
\text { promotora }(-308 \mathrm{G} / \mathrm{A}) \text { del gen }\end{array}$ \\
& ha sido también asociado con \\
& un incremento en la síntesis de \\
& TNF- $\alpha$ y con una mayor mortali- \\
& dad en pacientes con procesos \\
& infecciosos
\end{tabular}

TNF- $\beta \quad$ Linfocitos activados y fibroblastos, células endoteliales, células epiteliales y astrocitos

sTNFR-I y II Monocitos/macrófagos y linfo(CD120) citos T activas en respuesta a infecciones fúngicas

IL-1 $\quad$ Macrófagos activados

IL-1RA Miembro de la familia de la citocina IL-1 y es secretada por varios tipos de células incluyendo los adipocitos, células epiteliales y células inmunes

IL-6 Monocitos/macrófagos, fibroblastos, células endoteliales, linfocitos T y células del estroma de la médula ósea

IL-8 Todos los tipos de leucocitos, (quimiocina así como por otros muchos CXCL8) tipos celulares como: fibroblastos, células endoteliales, hepatocitos, astrocitos
En neutrófilos, induce la expresión de eROS y también es quimioatrayente. Incrementa la adhesión del endotelio

Receptores a TNF- $\alpha$ que disminuyen las concentraciones plasmáticas de la citocina

Mediador proinflamatorio que estimula la producción de una variedad a otras citocinas, como: IL-6, IL-8, MCP-1, MIP-1 $\alpha$

Participa en la regeneración epitelial y es un potente inductor de la fibrosis pulmonar

Inhibe la actividad de IL- $1 \alpha \mathrm{e}$ IL-1 $\beta$

Variante para el gen IL1RN (rs315952c)

Potente citocina proinflamatoria con una amplia variedad de funciones, pero específicamente está involucrada en la producción de fiebre y la maduración y la diferenciación de las células B

Los niveles de IL-6 en plasma están influenciados por el polimorfismo -174G/C

Desempeña un papel en la quimiotaxis

Variante genotípica-251A/T
No se ha encontrado asociación de los polimorfismos TNF- $\beta$ 1/2 con el pronóstico

Fuertemente asociados con el aumento de la morbilidad y la mortalidad

Es activa en las primeras etapas de la LPA. Adicionalmente induce la expresión de ICAM-1 y NOS2 mediante la activación de NF-kb para promover el reclutamiento de neutrófilos de la circulación durante LPA

Asociada con una reducción del riesgo y un marcado incremento en los niveles plasmáticos de IL1RA

Altos niveles de IL- 6 en el LBA o $\quad 11,16,17$ plasma son de mal pronóstico

Mayor susceptibilidad

En LBA de pacientes SDRA, los niveles de IL-8 aumentan considerablemente y muestran una elevada asociación para mayor riesgo. También los niveles de IL-8 en LBA se asociaron independientemente con mayor mortalidad

Los pacientes con este alelo aumentaron la síntesis de IL-8
9,10 


\section{Continuación de la Tabla $1 .$.}

IL-10 Linfocitos Th2, monocitos/macrófagos, linfocitos $B$, queratinocitos y otros tipos celulares

IL-13 Linfocitos Th2

CRP (proteína C En la fase aguda es sintetizada reactiva)

Procalcitonina Molécula inflamatoria que se relaciona con la CRP

MBL Hígado

(lectina de unión a manosa)
Comparte similaridad estructural y funcional con las proteínas del surfactante pulmonar A y $D$
MIF Linfocitos $T$, células del pán-

(factor inhibidor de la migración de macrófagos)
Posee actividad antiinflamatoria, incluida la represión de activación de los neutrófilos y disminuye la expresión de citocinas proinflamatorias, como el TNF- $\alpha$, la NOS2, la apoptosis y suprime la activación de macrófagos

Su expresión está regulada por un SNP en la región del promotor (-1082G/A)
Niveles elevados de IL-10 en

11,20 plasma se asocian con un mal pronóstico

EI SNP -1082G/G parece estar asociado con menor riesgo de insuficiencia respiratoria aguda en pacientes traumatizados, y se asocia con menores grados de severidad, disfunción orgánica y mortalidad

Inhibe producción de TNF- $\alpha$, IL$1 \beta$, IL-8, aumentando al mismo tiempo la producción de IL-1RA Inhibe la activación del NF-kb Altera la expresión de las proteínas del surfactante (SP-C y SP-D)

Altas concentraciones de IL-13, en plasma de pacientes con SDRA

$20,22,23$

Estimulada por la liberación de citocinas, como la IL-6

Se reconoce como parte de la respuesta inflamatoria sistémica a procesos sépticos ya que aumenta como respuesta a endotoxinas

Es capaz de unirse con azúcares presentes en una amplia variedad de bacterias. $Y$ otros microorganismos promoviendo su eliminación mediante la activación del complemento y participa en la fagocitosis por opsoninas

Cada uno de los tres SNPs reduce la cantidad de MBL de 5 a 10 veces exón 1:

Codón 52 (rs5030737;

Arg $\rightarrow$ Cys, alelo D)

Codón 54 (rs1800450;

Gly $\rightarrow$ Asp, alelo B)\} Codón 57 (rs1800451;

Gly $\rightarrow$ Glu, alelo C)

El SNP del codón -221

(rs7096202; tipo X/Y) disminuye significativa en la concentración sérica $M B L$

Citocina proinflamatoria con un papel importante en la respuesta a endotoxinas
No se ha determinado el valor diagnóstico diferencial

Marcador pronóstico útil de mortalidad

La presencia de polimorfismos en el gen MBL2 afecta la estructura e influencia las concentraciones séricas de MBL; y por lo tanto, se asocia con procesos infecciosos
El nivel de expresión de MIF y los niveles de macrófagos pueden ser un buen índice en el diagnóstico temprano 


\section{Continúa de la Tabla $1 . .$.}

PBEF Linfocitos activados en células (visfatina) estromales de la médula ósea. Recientemente identificado como una adipocina, debido a sus altos niveles de expresión en adipocitos

$N F-\kappa B$

Factor de transcripción clave en la regulación de varios genes de la respuesta proinflamatoria
Induce la expresión de citocinas Niveles altos de esta citocina inflamatorias tales como TNF- $\alpha$, IL-1 $\beta$ y IL-6

Regula la expresión de genes como: moléculas de adhesión (ICAM-1), enzimas (iNOS, ciclooxigenasa-2) y citocinas (IL$1 \beta$, TNF- $\alpha$, IL-6) y quimiocinas

La presencia del haplotipo GTC (-881G/-826T/-297C) del gen NF-KBIA

Polimorfismo en el promotor de NFKB1 (-94ins/delATTG o -24.219ins/delATTG) pueden encontrarse en la circulación sistémica de los pacientes con sepsis, lesión pulmonar agu$\mathrm{da}$, y otros procesos inflamatorios

El polimorfismo -1001T/G se asociada con un aumento en la susceptibilidad y mayor riesgo de mortalidad, mientras que el polimorfismo -1543C/T se asoció con un mejor pronóstico

29

Se asocia con un mayor riesgo de SDRA en caucásicos

Influye en la gravedad, pero no 33 el espacio aéreo alveolar requiere una barrera epitelial alveolar intacta, y pacientes con bajas tasas de drenado tienen una mayor duración de ventilación mecánica, mala oxigenación y mayor mortalidad en comparación con los pacientes que tienen tasas normales de drenaje. Los daños al epitelio alveolar son parte de los fenómenos observados en la LPA y su mayor comprensión puede ser de valor pronóstico en SDRA. Algunos marcadores potenciales de lesiones en el epitelio alveolar incluyen proteínas del surfactante (SP), así como glicoproteína $\mathrm{KL}-6$, todos los cuales son liberados de células epiteliales tipo II lesionadas. Las moléculas asociadas a estos fenómenos se muestran en la tabla 3.

\section{MARCADORES DE LESIÓN ENDOTELIAL}

El endotelio microvascular pulmonar es un órgano metabólicamente activo esencial para mantener la homeostasis pulmonar y cardiovascular. Los estímulos dañinos comprometen la integridad estructural y funcional endotelial pulmonar, y conducen a la formación de edema pulmonar no cardiogénico e inflamación parenquimal. Así, el endotelio pulmonar tiene un papel clave en el desarrollo de la LPA y el SDRA. Las moléculas que se asocian con alteraciones en el endotelio microvascular se muestran en la tabla 4.

\section{MECANISMOS ANTIOXIDANTES}

Se cree que las especies reactivas del oxígeno (ROS) juegan un papel muy importante en la patogenia_del SDRA, pero el mecanismo no está bien comprendido.

Los radicales de oxígeno $(\mathrm{RO})$ se producen en altas concentraciones bajo condiciones de isquemia-reperfusión. Para protegerse de sus efectos dañinos, las células producen varios tipos de sistemas antioxidantes. Dentro de los antioxidantes enzimáticos tenemos a la superóxido dismutasa (SOD), enzima que se expresa fuertemente en los pulmones y juega un papel importante como regulador de procesos inflamatorios y estrés oxidativo. El gen para SOD se presenta en el cromosoma 4 y la presencia de SNPs da como resultado el remplazo de una Arg por una Gly, lo cual produce un aumento de 10 veces en la actividad enzimática. Además de esta alteración, otros tres SNPs parecen estar asociados con el SDRA. Arcaroli et al., ${ }^{63}$ encontraron que en 179 pacientes caucásicos con el haplotipo GCCT, presentaron protección para el SDRA (tal vez por promover niveles elevados de SOD en pulmón) y una menor mortalidad.

Todas las evidencias sugieren que los ROS pueden desempeñar un papel clave en la fase de iniciación del SDRA, mientras que el anión superóxido puede ser un actor protagónico en este proceso, y SOD funcionar como un sistema protector de SDRA. 
Tabla 2. Marcadores relacionados a cambios en la permeabilidad y tono vascular en SDRA.

\begin{tabular}{|c|c|c|}
\hline Molécula & Fuente & Función \\
\hline TGF- $\alpha$ & $\begin{array}{l}\text { Miembro de la familia de pép- } \\
\text { tidos de factor de crecimiento } \\
\text { epidérmico (EGF). Es produ- } \\
\text { cida por macrófagos, células } \\
\text { del cerebro y queratinocitos }\end{array}$ & Induce desarrollo epitelial \\
\hline TGF- $\beta$ & $\begin{array}{l}\text { Producido por linfocitos } \mathrm{T} \text {, } \\
\text { plaquetas y otros muchos } \\
\text { tipos celulares. Presenta al } \\
\text { menos cinco isoformas } \\
\text { (TGF- } \beta 1-\beta 5 \text { ) }\end{array}$ & $\begin{array}{l}\text { Incrementa la proliferación } \\
\text { de fibroblastos, osteoblastos } \\
\text { y células de músculo liso e } \\
\text { incrementa la síntesis de la } \\
\text { matriz extracelular y es capaz } \\
\text { de inducir la fibroproliferación. } \\
\text { Inhibe la síntesis y/o el efecto } \\
\text { del IFN-gamma, TNF-alfa, } \\
\text { TNF-beta, IL-1, IL-2 e IL-3 }\end{array}$ \\
\hline \multirow[t]{2}{*}{ VEGF } & $\begin{array}{l}\text { Secretado por macrófagos, } \\
\text { plaquetas, queratinocitos, y } \\
\text { células mesangiales renales }\end{array}$ & \\
\hline & $\begin{array}{l}\text { También es secretada por cé- } \\
\text { lulas alveolares en respuesta } \\
\text { a una serie de estímulos } \\
\text { proinflamatorios }\end{array}$ & $\begin{array}{l}\text { Polimorfismo }+936 \mathrm{C} / \mathrm{T} \\
\text { (rs3025039) se ha relacio- } \\
\text { nado con bajos niveles de } \\
\text { VEGF en plasma, mientras } \\
\text { que el } 2460 \mathrm{C} / \mathrm{T} \text { (rs833061) y } \\
\text { los polimorfismos }+405 \mathrm{C} / \mathrm{G} \\
\text { (rs2010963) han demostrado } \\
\text { aumentar significativamente la } \\
\text { producción de VEGF }\end{array}$ \\
\hline
\end{tabular}

Ang-2 Producida por el endotelio (angiopoietina) y en menor concentración la Ang-2 es sintetizada por macrófagos

\section{ACE Altamente expresada en los} (convertasa de pulmones, donde degrada angiotensina) la bradiquinina y cataliza la formación de la angiotensina II (Ang II). Se encuentra en cantidades variables en la superficie de las células epiteliales y endoteliales del pulmón
Reguladores de permeabilidad vascular

Polimorfismos de Angpt-2 parecen asociarse con niveles más altos de Ang-2 en el plasma Aumento de la permeabilidad vascular

Polimorfismo funcional, consiste en una inserción (I) o deleción (D) de un fragmento de $287 \mathrm{bp}$ en el intrón 16 que determina tres genotipos (DD, ID y II) La presencia del alelo $D$ se asocia con una mayor actividad de ACE en circulación y tejido y, consecuentemente, con niveles significativamente mayores de Ang II y menores de bradiquinina
Asociación con LPA/SDRA

Ref.

Niveles elevados en LBA de pacientes con SDRA

34

En experimentos in vitro, se ha mostrado que TGF- $\alpha$ induce reparación del epitelio alveolar

Con relevancia no sólo en las fases tempranas de la lesión pulmonar aguda, sino también afecta el funcionamiento del drenado de líquido alveolar al interferir con las vías de señalización que conducen a la activación del canal de sodio (ENaC)

Los niveles de VEGF aumentan en plasma y se reducen en el intersticio pulmonar

En el polimorfismo $+936 \mathrm{C} / \mathrm{T}$, las frecuencias de los genotipos CT y TT estaban aumentadas en los pacientes con SDRA, y se asocian significativamente con aumento en la mortalidad

Asociación positiva entre altas concentraciones de Ang-2 en plasma y SDRA inducida por sepsias

Niveles más altos de Ang-2 en los pacientes que desarrollaron SDRA

Frecuencia de genotipo DD aumentado en los pacientes con SDRA El polimorfismo I/D no se asocia con susceptibilidad a LPA/SDRA, pero se asocia con el riesgo de mortalidad en población asiática 


\section{Continuación de la Tabla $2 .$.}

ACE2 Se expresa abundantemente (convertasa de en las células Clara, monoangiotensina 2) citos tipo I y II, macrófagos, endotelio y epitelio bronquial

\section{MYLK}

(cinasa de cadena ligera de miosina)

\section{Es una serina/treonina} proteína quinasa específica que fosforila la cadena ligera reguladora de la miosina II. Existen cuatro isoformas diferentes:

MYLK - músculo liso

MYLK2 - esquelético

MYLK3 - cardíaca

MYLK4 - reciente
Es homólogo de ACE y funciona En pacientes con SDRA hay como un regulador negativo del un aumento en la actividad de sistema renina-angiotensina la ACE y una disminución de la actividad de ACE2

Relacionada con el incremento Evidencia sólida para el papel de en la permeabilidad del endote- MLCK en la patogenicidad y la lio vascular pulmonar; también susceptibilidad del SDRA es esencial para el movimiento de neutrófilos

Un polimorfismo localizado en la Una asociación significativa para región reguladora 5' del gen fue riesgo alto de desarrollar LPA encontrada para los haplotipos

EA y $A A$

Tabla 3. Marcadores relacionados con la tensión superficial, quimiotaxis y motilidad celular.

\begin{tabular}{|c|c|c|c|c|}
\hline Molécula & Fuente & Función & Asociación con LPA/SDRA & Ref. \\
\hline Surfactante pulmonar & Neumocitos tipo II & $\begin{array}{l}\text { Complejo de lípidos y } \\
\text { proteínas capaz de reducir } \\
\text { significativamente la tensión } \\
\text { superficial dentro de los } \\
\text { alvéolos pulmonares }\end{array}$ & & \\
\hline \multirow[t]{2}{*}{ SP-A y SP-D } & $\begin{array}{l}\text { Moléculas hidrofílicas de } \\
\text { alto peso molecular con } \\
\text { papeles marcados en inmu- } \\
\text { nidad innata }\end{array}$ & & $\begin{array}{l}\text { Se encontraron niveles bajos } \\
\text { de SP-A en LBA con un valor } \\
\text { predictivo negativo del } 100 \% \text {. } \\
\text { Elevados niveles en plasma } \\
\text { de SP-A en pacientes con } \\
\text { riesgo de SDRA secundario } \\
\text { a sepsis y bronco aspiración, } \\
\text { pero no en traumatismo grave }\end{array}$ & 48,49 \\
\hline & & & $\begin{array}{l}\text { Niveles plasmáticos eleva- } \\
\text { dos de SP-D se asocian con } \\
\text { un aumento del riesgo para } \\
\text { desarrollar LPA }\end{array}$ & \\
\hline SP-B y SP-C & $\begin{array}{l}\text { Son especies hidrofóbicas } \\
\text { de bajo peso molecular } \\
\text { esenciales para la inte- } \\
\text { gridad de la membrana } \\
\text { epitelial alveolar }\end{array}$ & 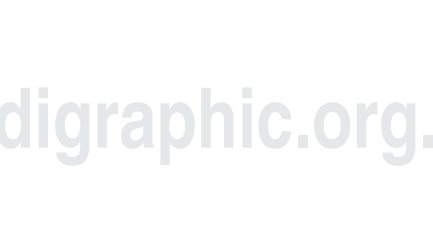 & $\begin{array}{l}\text { Variación alélica - } 1580 \mathrm{C} / \mathrm{T} \text { del } \\
\text { exón } 4 \text { del gen que codifica la } \\
\text { proteína SP-B, se asocia con } \\
\text { una mayor susceptibilidad a } \\
\text { SDRA en mujeres pero no en } \\
\text { hombres }\end{array}$ & 50,51 \\
\hline $\begin{array}{l}\text { Glicoproteína KL-6 } \\
\text { (Krebs von den Lun- } \\
\text { gen-6) }\end{array}$ & $\begin{array}{l}\text { El tipo de mucina es expre- } \\
\text { sado en los neumocitos tipo } \\
\text { II y por células epiteliales } \\
\text { bronquiales }\end{array}$ & $\begin{array}{l}\text { Sus niveles en plasma } \\
\text { correlacionan con el índice } \\
\text { de daño epitelio alveolar y } \\
\text { permeabilidad alvéolo capilar }\end{array}$ & $\begin{array}{l}\text { El marcador es inespecífico. } \\
\text { Los niveles plasmáticos KL-6 } \\
\text { son superiores en pacientes } \\
\text { que fallecen por SDRA }\end{array}$ & 52,53 \\
\hline
\end{tabular}


Tabla 4. Marcadores relacionados con la lesión endotelial.

\begin{tabular}{|c|c|c|c|c|}
\hline Molécula & Fuente & Función & Asociación con LPA/SDRA & Ref. \\
\hline $\begin{array}{l}\text { CYB5R3 } \\
\text { (citocromo-b5 reduc- } \\
\text { tasa) }\end{array}$ & $\begin{array}{l}\text { En los eritrocitos está pre- } \\
\text { sente como una isoforma } \\
\text { soluble que funciona para } \\
\text { reducir la metahemoglobi- } \\
\text { na a hemoglobina }\end{array}$ & $\begin{array}{l}\text { Sirve como un donador de } \\
\text { electrones para el sistema } \\
\text { del citocromo } \mathrm{P}_{450} \\
\text { Tiene un impacto directo so- } \\
\text { bre el estado de la cascada } \\
\text { inflamatoria }\end{array}$ & $\begin{array}{l}\text { La expresión disminuye signifi- } \\
\text { cativamente desde las primeras } \\
\text { etapas de la lesión pulmonar. } \\
\text { A la fecha no existen más } \\
\text { evidencias clínicas que asocien } \\
\text { al citocromo b5 con un valor de } \\
\text { pronóstico para LPA/SDRA }\end{array}$ & 54 \\
\hline
\end{tabular}

RAGE

\section{SCGB1A1}

(secretoglobina; proteína de células Claras;CC16)
Es una proteína transmembranal de la superfamilia de inmunoglobulinas y receptor multiligando que une glicoproteínas modificadas (incluyendo proteína HMGB-1). Aunque tiene una expresión ubicua, sus niveles de expresión son mayores en pulmón

\section{Secretadas por las células} Clara cuando son alteradas por un daño pulmonar inducido por agentes tóxicos
La interacción entre RAGE Es un marcador específico de y sus ligandos resulta en la daño epitelial pulmonar. Tiene activación de genes proinfla- capacidad de diagnóstico para matorios

LPA y es factor pronóstico en pacientes que desarrollan SDRA por lesión pulmonar ventilatoria
Funciona como un potente quimioatrayente de neutrófilos y como inhibidor de la fosfatasa $A 2$

Un polimorfismo funcional $-26 \mathrm{G}>\mathrm{A}$
Desempeña un papel importante en la patofisiología del SDRA Hay resultados contradictorios sobre la capacidad de SCGB1A1 para diagnosticar con precisión LPA/SDRA

Relacionado con la disminución de los niveles de esta proteína en suero

Asociación con este polimorfismo con susceptibilidad a SDRA

PI3, elafin (inhibidor de peptidasa 3)

Inhibidor de proteasa producido localmente en el pulmón
Juega un papel central en el control del exceso de actividad elastasa

Tres variantes genéticas (rs19836491, rs6032040, y rs2664581)
La expresión de PI3 disminuye a medida que se reduce la gravedad del SDRA

El alelo rs2664581 (T34P) tiene una asociación significativa con mayor riesgo de SDRA. Adicionalmente, homocigóticos para un alelo A de rs2664581 mostraron niveles plasmáticos significativamente inferiores $(p$ $=0.019$ ) en comparación con homocigóticos o heterocigóticos para el alelo variante $\mathrm{C}$
Las anormalidades de la química del hierro en plasma y pulmón también han sido demostradas en SDRA. El hierro es un catalizador biológico clave para la utilización de oxígeno, también puede inducir la formación de productos intermedios reactivos y dañinos. Existen mecanismos de protección para limitar la toxicidad de hierro, pero los pacientes con SDRA presentan una disminución en la protección antioxidante contra el daño oxidativo catalizado por hierro.

La hemooxigenasa $(\mathrm{HO})$ es la principal enzima implicada en el catabolismo del grupo hemo. Se conocen tres isoformas de hemooxigenasa: $\mathrm{HO}-1, \mathrm{HO}-2, \mathrm{HO}-$ 3. La HO-1 es una forma inducible; normalmente se expresa en niveles bajos en la mayoría de los tejidos 
(incluyendo neumocitos tipo Il y macrófagos alveolares), pero su expresión es inducida por distintos estímulos fisiopatológicos como: la hipoxia, hiperoxia, óxido nítrico (NO), endotoxinas y citocinas proinflamatorias como IL-6 y TNF- $\alpha$. Esta enzima es conocida como citoprotectora ya que cataliza la degradación del grupo hemo (un potente oxidante) para generar biliverdina$\mathrm{IX} \alpha$, un ión ferroso $\left(\mathrm{Fe}^{+}\right)$y monóxido de carbono (CO). Biliverdina-IX $\alpha$ se convierte en bilirrubina-IX $\alpha$, que es un potente antioxidante endógeno, con propiedades antiinflamatorias recién reconocidas, mientras que el $\mathrm{Fe}^{+}$es secuestrado por ferritina, que posee efectos antiapoptóticos y antioxidantes adicionales. El CO tiene numerosas funciones biológicas, incluyendo propiedades antiinflamatorias y comparte muchas similitudes con el NO, como su capacidad para inhibir la proliferación de células de músculo liso y la agregación plaquetaria, así como capacidad para modular el tono vascular, aumentando los niveles de GMPc. ${ }^{64}$

Los niveles de HO-1 se incrementan en varias enfermedades pulmonares como el SDRA, asma, fibrosis quística, fibrosis idiopática y en el rechazo o trasplante pulmonar, y juega un papel fundamental en la defensa del pulmón contra la respuesta inflamatoria, el estrés oxidativo y la lesión tisular, por lo que se le considera como un citoprotector en la LPA. ${ }^{65}$

Además de sus propiedades antioxidantes y antiinflamatorias, datos recientes sugieren que $\mathrm{HO}-1$ puede modular la expresión de la proteína surfactante B (SP-B) durante el SDRA. ${ }^{66}$ En un modelo de ratón de SDRA inducido por nebulización con endotoxina, ratones deficientes en $\mathrm{HO}-1$ desarrollaron graves daños fisiológicos en el pulmón, que correlacionan con una marcada reducción en los niveles de SP-B. ${ }^{64}$ En otros estudios, la inducción de HO-1 también ha mostrado ser un factor de mejor pronóstico en animales con LPA inducida por lipopolisacárido (LPS), sugiriendo que esta enzima puede ser un blanco para activar su función en el tratamiento de pacientes con LPA/SDRA.

En un estudio retrospectivo de Mumby et al., encontraron que las concentraciones de HO-1 en lavado bronquioalveolar (LBA) de pacientes con SDRA, se correlacionan significativamente con los índices de la movilización del hierro. ${ }^{67}$ La inducción de HO-1 también ejerce funciones inmunomoduladoras en pacientes con LPA, y recientemente se ha encontrado que la activación PPARy (receptor activado por el proliferador de peroxisoma) es responsable de inducir la expresión de HO-1. Así, se propone que la activación del PPARy también puede ser una estrategia terapéutica en pacientes con LPA/SDRA. ${ }^{68}$

Además, existe un polimorfismo en la región del promotor de HO-1 (gen: HMOX1) que provoca una re- ducción en la expresión de la enzima, disminuyendo la capacidad citoprotectora, lo que da como resultado un mayor riesgo de enfermedades respiratorias. La variante más estudiada es el polimorfismo de dinucleótido de repetición [GT]n, localizado en aproximadamente -200 pb de la región proximal del promotor. La longitud del dinucleótido oscila entre $[G T]_{10}[G T]_{40}$ y representa alelos continuos. ${ }^{69}$ En un estudio con 437 pacientes caucásicos se encontró que el alelo $L(G T>31)$ correlacionó con un aumento en los niveles plasmáticos de HO-1 y se asoció con riesgo reducido a SDRA. ${ }^{70}$

\section{FACTORES RELACIONADOS CON LA COAGULA- CIÓN Y FIBRINÓLISIS}

Además de los marcadores inflamatorios, aquellos que regulan la coagulación y la fibrinólisis parecen ser también marcadores predictivos en el resultado clínico en pacientes con LPA/SDRA. Y es que la activación de la célula endotelial y de la plaqueta puede conducir a una coagulopatía en pacientes con sepsis o SDRA. ${ }^{71}$

La fibrinólisis (proceso de disolución de fibrina por proteasas sanguíneas), también se encuentra alterado en pacientes con LPA/SDRA. Durante la fase exudativa del SDRA, se presenta un desequilibrio entre los procesos de activación de la coagulación y la activación de fibrinólisis. Se producen abundantes depósitos de fibrina bronquioloalveolar y fibronectina, los cuales promueven la formación de membrana hialina y posterior fibrosis alveolar. Moléculas implicadas en estos procesos se muestran en el tabla 5.

\section{CONCLUSIÓN}

Debido a que la LPA/SDRA es una condición que puede ocurrir como resultado de múltiples enfermedades, es probable que la fisiopatología que lleva a la falla pulmonar pueda ser muy diferente, a pesar del hecho de que un estado final común puede estar presente. En este sentido, diferenciar el LPA/SDRA basado en la condición subyacente ha sido ampliamente rechazado. Esta heterogeneidad parece ser la principal razón por la que difieren los resultados de la investigación básica de LPA y porqué al mismo tiempo, los ensayos clínicos a menudo no logran mostrar mejora en la supervivencia global, en varios parámetros fisiológicos o incluso la supervivencia de subgrupos específicos.

Más estudios de investigación básica con fenotipos más precisos que permitan disminuir la heterogeneidad de pacientes con LPA/SDRA deberían ser abordados. Éstos permitirán detallar los mecanismos fisiológicos, moleculares y celulares que protegen al pulmón de 
Tabla 5. Marcadores relacionados a la coagulación.

\begin{tabular}{lll}
\hline Molécula & \multicolumn{1}{c}{ Fuente } & \multicolumn{1}{c}{ Función } \\
\hline La proteína C activada & Es un inhibidor de la casca- & Otras funciones asociadas \\
(APC; autoprotrombina & da de coagulación y previe- & a la APC son: la supresión \\
ne la formación de coágulos & de interleucinas proinfla- \\
& mediante la inactivación de & matorias, la adherencia de \\
& los factores Va y VIIla & leucocitos al endotelio y la \\
& & inhibición de la apoptosis \\
& mediada por p53. La APC \\
& también puede neutralizar \\
& PAI-I y promover la fibrinó- \\
& losis
\end{tabular}

FVL

(factor V Leiden)

vWF

(factor de von Willebrand)

Urocinasa

PAI-1

(inhibidor del activador del plasminógeno-1)
Una substitución del aminoácido (Arg506Gln) en el factor $V$ de coagulación causa resistencia a la APC (APCR)

Es una glicoproteína multimérica implicadas en la hemostasia. Se sintetiza principalmente en las células endoteliales y en menor medida en las plaquetas

Existen dos formas de urocinasa: uPA y tPA Producido por células endoteliales activadas
Incrementa los niveles de trombina

Realiza funciones duales como la unión de plaquetas al endotelio y como una proteína de transporte para el factor VIII

La UPA es una serin-proteasa que activa el plasminógeno para formar la plasmina. Participa en la trombólisis o degradación de la matriz extracelular. Adicionalmente, la uPA potencia la activación de neutrófilos El polimorfismos del gene de UPA es: haplotipo CGCCCC

Expresado por células endoteliales y otras células inflamatorias

Regula la actividad de: uPA y tPA y contribuye a la remodelación tisular
Asociación con LPA/SDRA

Ref.

Existe una fuerte asociación

72,73

entre los niveles bajos de

APC en plasma, con una

alta mortalidad y mayor

dependencia de ayuda

ventilatoria y para pacientes

que desarrollan SDRA. En

muchos de estos casos,

los niveles de trombomo-

dulina en plasma o espacio

alveolar son elevados y

correlacionan con un mal

pronóstico

Pacientes heterocigotos para Arg506Gln del FVL presentan un aumento en la sobrevida a 30 días en comparación a los homocigotos para este gen

Su utilidad no ha sido aún completamente validada Un aumento de vWF en el suero y LBA de pacientes con LPA, correlaciona con mayor tasa de mortalidad y menos días de ventilación no asistida

Concentraciones elevadas

de la enzima contribuyen a la severidad del cuadro Una actividad disminuida de uroquinasa en LBA de pacientes con LPA/SDRA, se

asocia con niveles elevados de PAI-1

Presenta una fuerte asociación estadística con el índice de mortalidad a 60 días y días libres con ayuda ventilatoria

74,75

$71,76,77$

78
La elevación se asocia con un aumento de la mortalidad y más días de ayuda ventilatoria lesiones y estrés mecánico y que potencialmente modulan la inflamación sistémica.

Entender los marcadores bioquímicos y biológicos implicados en la respuesta compleja e inflamatoria del SDRA ofrece la posibilidad de futuras investigaciones al tratamiento objetivo de estos mediadores para predecir con mayor precisión el inicio o el resultado de la lesión que se produce en los pulmones. Métodos celulares y moleculares combinados con modelos animales y estudios clínicos pueden conducir a nuevos avances en la detección y el tratamiento del SDRA. 


\section{REFERENCIAS}

1. Donahoe M. Acute respiratory distress syndrome: A clinical review. Pulm Circ 2011;1(2):192-211. doi: 10.4103/2045-8932.83454.

2. Rubenfeld GD, Herridge MS. Epidemiology and outcomes of acute lung injury. Chest 2007;131(2):554562.

3. Walkey AJ, Summer R, Ho V, Alkana P. Acute respiratory distress syndrome: epidemiology and management approaches. Clin Epidemiol 2012;4:159-169. doi: 10.2147/ CLEP.S28800.

4. ARDS Definition Task Force; Ranieri VM, Rubenfeld GD, Thompson BT, et al. Acute respiratory distress syndrome: the Berlin definition. JAMA 2012;307(23):25262533. doi: 10.1001/jama.2012.5669.

5. Fanelli V, Vlachou A, Ghannadian S, Simonetti U, Slutsky AS, Zhang H. Acute respiratory distress syndrome: new definition, current and future therapeutic options. J Thorac Dis 2013;5(3):326-334. doi: 10.3978/j.issn.20721439.2013.04.05.

6. Pierrakos C, Karanikolas M, Scolletta S, Karamouzos V, Velissaris D. Acute respiratory distress syndrome: pathophysiology and therapeutic options. J Clin Med Res 2012;4(1):7-16. doi: 10.4021/jocmr761w.

7. Nader ND, McQuiller PS, Raghavendran K, Spengler $\mathrm{RN}$, Knight PR. The role of alveolar macrophages in the pathogenesis of aspiration pneumonitis. Immunol Invest 2007;36(4):457-471.

8. Ware LB. Pathophysiology of acute lung injury and the acute respiratory distress syndrome. Semin Respir Crit Care Med 2006;27(4):337-349.

9. Teuffel O, Ethier MC, Beyene J, Sung L. Association between tumor necrosis factor-alpha promoter -308 A/G polymorphism and susceptibility to sepsis and sepsis mortality: a systematic review and meta-analysis. Crit Care Med 2010;38(1):276-282. doi: 10.1097/ CCM.0b013e3181b42af0.

10. Gong MN, Zhou W, Williams PL, et al. -308GA and TNFB polymorphisms in acute respiratory distress syndrome. Eur Respir J 2005;26(3):382-389.

11. Parsons PE, Eisner MD, Thompson BT, et al.; NHLBI Acute Respiratory Distress Syndrome Clinical Trials Network. Lower tidal volume ventilation and plasma cytokine markers of inflammation in patients with acute lung injury. Crit Care Med 2005;33(1):1-6.

12. Olman MA, White KE, Ware LB, et al. Pulmonary edema fluid from patients with early lung injury stimulates fibroblast proliferation through IL-1 beta-induced IL-6 expression. J Immunol 2004;172(4):2668-2677.

13. Ganter MT, Roux J, Miyazawa B, et al. Interleukin1 beta causes acute lung injury via alphavbeta5 and alphavbeta 6 integrin-dependent mechanisms. Circ Res 2008;102(7):804-812. doi: 10.1161/CIRCRESAHA.107.161067.

14. Park WY, Goodman RB, Steinberg KP, et al. Cytokine balance in the lungs of patients with acute respiratory distress syndrome. Am J Respir Crit Care Med 2001;164(10 Pt 1):1896-1903.
15. Meyer NJ, Feng $\mathrm{R}$, Li M, et al. IL1RN coding variant is associated with lower risk of acute respiratory distress syndrome and increased plasma IL-1 receptor antagonist. Am J Respir Crit Care Med 2013;187(9):950-959. doi: 10.1164/rccm.201208-15010C.

16. McClintock D, Zhuo H, Wickersham N, Matthay MA, Ware LB. Biomarkers of inflammation, coagulation and fibrinolysis predict mortality in acute lung injury. Crit Care 2008;12(2):R41. doi: 10.1186/cc6846.

17. Lin WC, Lin CF, Chen CL, Chen CW, Lin YS. Prediction of outcome in patients with acute respiratory distress syndrome by bronchoalveolar lavage inflammatory mediators. Exp Biol Med (Maywood) 2010;235(1):57-65. doi: 10.1258/ebm.2009.009256.

18. Flores C, Ma SF, Maresso K, Wade MS, Villar J, Garcia JG. IL6 gene-wide haplotype is associated with susceptibility to acute lung injury. Transl Res 2008;152(1):11-17. doi: 10.1016/j.trsl.2008.05.006.

19. Mukaida N. Pathophysiological roles of interleukin-8/ CXCL8 in pulmonary diseases. Am J Physiol Lung Cell Mol Physiol 2003;284(4):L566-577.

20. Schottelius AJ, Mayo MW, Sartor RB, Baldwin AS Jr. Interleukin-10 signaling blocks inhibitor of kappa $B$ kinase activity and nuclear factor kappa B DNA binding. J Biol Chem 1999;274(45):31868-31874.

21. Gong MN, Thompson BT, Williams PL, et al. Interleukin-10 polymorphism in position -1082 and acute respiratory distress syndrome. Eur Respir J 2006;27(4):674681.

22. Cross LJ, Matthay MA. Biomarkers in acute lung injury: insights into the pathogenesis of acute lung injury. Crit Care Clin 2011;27(2):355-377. doi: 10.1016/j. ccc.2010.12.005.

23. Ito Y, Mason RJ. The effect of interleukin-13 (IL-13) and interferon- $\gamma$ (IFN- $\gamma$ ) on expression of surfactant proteins in adult human alveolar type // cells in vitro. Respir Res 2010;11:157. doi: 10.1186/1465-9921-11-157.

24. Bajwa EK, Khan UA, Januzzi JL, Gong MN, Thompson BT, Christiani DC. Plasma C-reactive protein levels are associated with improved outcome in ARDS. Chest 2009;136(2):471-480. doi: 10.1378/chest.08-2413.

25. Tseng JS, Chan MC, Hsu JY, Kuo BI, Wu CL. Procalcitonin is a valuable prognostic marker in ARDS caused by community-acquired pneumonia. Respirology 2008;13(4):505-509. doi: 10.1111/j.14401843.2008.01293.x.

26. Turner MW. The role of mannose-binding lectin in health and disease. Mol Immunol 2003;40(7):423-429.

27. Garred P, Pressler T, Madsen HO, et al. Association of mannose-binding lectin gene heterogeneity with severity of lung disease and survival in cystic fibrosis. J Clin Invest 1999;104(4):431-437.

28. Gao L, Flores C, Fan-Ma S, et al. Macrophage migration inhibitory factor in acute lung injury: expression, biomarker, and associations. Transl Res 2007;150(1):18-29.

29. Luk T, Malam Z, Marshall JC. Pre-B cell colony-enhancing factor (PBEF)/visfatin: a novel mediator of innate immunity. J Leukoc Biol 2008;83(84):804-816. doi: 10.1189/ jlb.0807581. 
30. Bajwa EK, Yu CL, Gong MN, Thompson BT, Christiani DC. Pre-B-cell colony-enhancing factor gene polymorphisms and risk of acute respiratory distress syndrome. Crit Care Med 2007;35(5):1290-1295.

31. Christman JW, Sadikot RT, Blackwell TS. The role of nuclear factor-kappa $B$ in pulmonary diseases. Chest 2000;117(5):1482-1487.

32. Adamzik M, Frey UH, Rieman K, et al. Insertion/deletion polymorphism in the promoter of NFKB1 influences severity but not mortality of acute respiratory distress syndrome. Intensive Care Med 2007;33(7):1199-1203.

33. Zhai R, Zhou W, Gong MN, et al. Inhibitor kappa B-alpha haplotype GTC is associated with susceptibility to acute respiratory distress syndrome in Caucasians. Crit Care Med 2007;35(3):893-898.

34. Madtes DK, Rubenfeld G, Klima LD, et al. Elevated transforming growth factor-alpha levels in bronchoalveolar lavage fluid of patients with acute respiratory distress syndrome. Am J Respir Crit Care Med 1998;158(2):424430.

35. Peters DM, Vadász I, Wujak L, et al. TGF- $\beta$ directs trafficking of the epithelial sodium channel ENaC which has implications for ion and fluid transport in acute lung injury. Proc Natl Acad Sci USA 2014;111(3):E374-383. doi: 10.1073/pnas.1306798111

36. Frank JA, Matthay MA. TGF- $\beta$ and lung fluid balance in ARDS. Proc Natl Acad Sci USA 2014;111(3):885-886. doi: 10.1073/pnas.1322478111.

37. Medford AR, Millar AB. Vascular endothelial growth factor (VEGF) in acute lung injury (ALI) and acute respiratory distress syndrome (ARDS): paradox or paradigm? Thorax 2006;61(7):621-626.

38. Zhai R, Gong MN, Zhou W, et al. Genotypes and haplotypes of the VEGF gene are associated with higher mortality and lower VEGF plasma levels in patients with ARDS. Thorax 2007;62(8):718-722.

39. Fiedler U, Scharpfenecker M, Koidl S, et al. The Tie-2 ligand angiopoietin-2 is stored in and rapidly released upon stimulation from endothelial cell Weibel-Palade bodies. Blood 2004;103(11):4150-4156.

40. Gallagher DC, Parikh SM, Balonov K, et al. Circulating angiopoietin 2 correlates with mortality in a surgical population with acute lung injury/adult respiratory distress syndrome. Shock 2008;29(6):656-661.

41. Meyer NJ, Li M, Feng R, et al. ANGPT2 genetic variant is associated with trauma-associated acute lung injury and altered plasma angiopoietin-2 isoform ratio. Am J Respir Crit Care Med 2011;183(10):1344-1353. doi: 10.1164/rccm.201005-07010C.

42. Marshall RP, Webb S, Bellingan GJ, et al. Angiotensin converting enzyme insertion/deletion polymorphism is associated with susceptibility and outcome in acute respiratory distress syndrome. Am J Respir Crit Care Med 2002;166(5):646-650.

43. Matsuda A, Kishi T, Jacob A, Aziz M, Wang P. Association between insertion/deletion polymorphism in angiotensin-converting enzyme gene and acute lung injury/acute respiratory distress syndrome: a metaanalysis. BMC Med Genet 2012;13:76.
44. Imai Y, Kuba K, Penninger JM. Angiotensin-converting enzyme 2 in acute respiratory distress syndrome. Cell Mol Life Sci 2007;64(15):2006-2012.

45. Wösten-van Asperen RM, Bos AP, Bem RA, et al. Imbalance between pulmonary angiotensin-converting enzyme and angiotensin-converting enzyme 2 activity in acute respiratory distress syndrome. Pediatr Crit Care Med 2013;14(9):e438-441. doi: 10.1097/ PCC.0b013e3182a55735.

46. Christie JD, Ma SF, Aplenc R, et al. Variation in the myosin light chain kinase gene is associated with development of acute lung injury after major trauma. Crit Care Med 2008;36(10):2794-2800.

47. Gao L, Grant A, Halder I, et al. Novel polymorphisms in the myosin light chain kinase gene confer risk for acute lung injury. Am J Respir Cell Mol Biol 2006;34(4):487495.

48. McCormack FX, Gibbons R, Ward SR, Kuzmenko A, Wu H, Deepe GS Jr. Macrophage-independent fungicidal action of the pulmonary collections. J Biol Chem 2003;278(38):36250-36256.

49. Eisner MD, Parsons P, Matthay MA, Ware L, Greene K; Acute Respiratory Distress Syndrome Network. Plasma surfactant protein levels and clinical outcomes in patients with acute lung injury. Thorax 2003;58(11):983988.

50. Gong MN, Wei Z, Xu LL, Miller DP, Thompson BT, Christiani DC. Polymorphism in the surfactant protein- $B$ gene, gender, and the risk of direct pulmonary injury and ARDS. Chest 2004;125(1):203-211.

51. Quasney MW, Waterer GW, Dahmer MK, et al. Association between surfactant protein $B+1580$ polymorphism and the risk of respiratory failure in adults with community-acquired pneumonia. Crit Care Med 2004;32(5):1115-1119.

52. Sato $\mathrm{H}$, Callister ME, Mumby $\mathrm{S}$, et al. KL-6 levels are elevated in plasma from patients with acute respiratory distress syndrome. Eur Respir J 2004;23(1):142-145.

53. Ishizaka A, Matsuda T, Albertine $\mathrm{KH}$, et al. Elevation of $K L-6$, a lung epithelial cell marker, in plasma and epithelial lining fluid in acute respiratory distress syndrome. Am J Physiol Lung Cell Mol Physiol 2004;286(6):L10881094.

54. Ménoret A, Kumar S, Vella AT. Cytochrome b5 and cytokeratin 17 are biomarkers in bronchoalveolar fluid signifying onset of acute lung injury. PLoS One 2012;7(7):e40184. doi: 10.1371/journal.pone.0040184.

55. Creagh-Brown BC, Quinlan GJ, Evans TW, Burke-Gaffney A. The RAGE axis in systemic inflammation, acute lung injury and myocardial dysfunction: an important therapeutic target? Intensive Care Med 2010;36(19):16441656. doi: 10.1007/s00134-010-1952-z.

56. Fremont RD, Koyama T, Calfee CS, et al. Acute lung injury in patients with traumatic injuries: utility of a panel of biomarkers for diagnosis and pathogenesis. J Trauma 2010;68:1121-1127. doi: 10.1097/TA.0b013e3181c40728.

57. Determann RM, Millo JL, Waddy S, Lutter R, Garrard CS, Schultz MJ. Plasma CC16 levels are associated with development of ALI/ARDS in patients with ventilator- 
associated pneumonia: a retrospective observational study. BMC Pulm Med 2009;9:49. doi: 10.1186/14712466-9-49.

58. Kropski JA, Fremont RD, Calfee CS, Ware LB. Clara cell protein (CC16), a marker of lung epithelial injury, is decreased in plasma and pulmonary edema fluid from patients with acute lung injury. Chest 2009;135(6):14401447. doi: 10.1378/chest.08-2465.

59. Frerking I, Sengler C, Günther A, et al. Evaluation of the -26G > A CC16 polymorphism in acute respiratory distress syndrome. Crit Care Med 2005;33(10):2404-2406.

60. Wang Z, Beach D, Su L, Zhai R, Christiani DC. A genomewide expression analysis in blood identifies pre-elafin as a biomarker in ARDS. Am J Respir Cell Mol Biol 2008;38(6):724-732. doi: 10.1165/rcmb.2007-03540C.

61. Wang Z, Chen F, Zhai R, et al. Plasma neutrophil elastase and elafin imbalance is associated with acute respiratory distress syndrome (ARDS) development. PLoS One 2009;4(2):e4380. doi: 10.1371/journal. pone.0004380.

62. Tejera P, Wang Z, Zhai R, et al. Genetic polymorphisms of peptidase inhibitor 3 (elafin) are associated with acute respiratory distress syndrome. Am J Respir Cell Mol Biol 2009;41(6):696-704. doi: 10.1165/rcmb.2008-04100C.

63. Arcaroli JJ, Hokanson JE, Abraham E, et al. Extracellular superoxide dismutase haplotypes are associated with acute lung injury and mortality. Am J Respir Crit Care Med 2009;179(2):105-112. doi: 10.1164/rccm.20071015660 C

64. Fredenburgh LE, Baron RM, Carvajal IM, et al. Absence of heme oxygenase-1 expression in the lung parenchyma exacerbates endotoxin-induced acute lung injury and decreases surfactant protein- $B$ levels. Cell Mol Biol (Noisy-le-grand) 2005;51(5):513-520.

65. Fredenburgh LE, Perrella MA, Mitsialis SA. The role of heme oxygenase-1 in pulmonary disease. Am J Respir Cell Mol Biol 2007;36(2):158-165.

66. Adamzik M, Frey U, Sixt S, et al. ACE I/D but not AGT (-6)A/G polymorphism is a risk factor for mortality in ARDS. Eur Respir J 2007;29(3):482-488.

67. Mumby S, Upton RL, Chen Y, et al. Lung heme oxygenase-1 is elevated in acute respiratory distress syndrome. Crit Care Med 2004;32(5):1130-1135.

68. Wang G, Han D, Zhang Y, et al. A novel hypothesis: up-regulation of HO-1 by activation of PPAR inhibits HMGB1-RAGE signaling pathway and ameliorates the development of ALI/ARDS. J Thorac Dis 2013;5(5):706710. doi: 10.3978/j.issn.2072-1439.2013.08.69.

69. Exner M, Minar E, Wagner O, Schillinger M. The role of heme oxygenase-1 promoter polymorphisms in human disease. Free Radic Biol Med 2004;37(8):1097-1104.

70. Sheu CC, Zhai R, Wang Z, et al. Heme oxygenase-1 microsatellite polymorphism and haplotypes are associated with the development of acute respiratory distress syndrome. Intensive Care Med 2009;35(8):1343-1351. doi: 10.1007/s00134-009-1504-6.

71. Flori HR, Ware LB, Milet M, Matthay MA. Early elevation of plasma von Willebrand factor antigen in pediatric acute lung injury is associated with an increased risk of death and prolonged mechanical ventilation. Pediatr Crit Care Med 2007;8(2):96-101.

72. Ware LB, Fang X, Matthay MA. Protein C and thrombomodulin in human acute lung injury. Am J Physiol Lung Cell Mol Physiol 2003;285(3):L514-L521.

73. Yan SB, Helterbrand JD, Hartman DL, Wright TJ, Bernard GR. Low levels of protein C are associated with poor outcome in severe sepsis. Chest 2001;120(3):915-922.

74. Adamzik M, Frey UH, Riemann K, et al. Factor V Leiden mutation is associated with improved 30-day survival in patients with acute respiratory distress syndrome. Crit Care Med 2008;36(6):1776-1779. doi: 10.1097/ CCM.0b013e318174373d.

75. Benfield T, Ejrnaes K, Juul K, et al. Influence of factor $V$ Leiden on susceptibility to and outcome from critical illness: a genetic association study. Crit Care 2010;14(2):R28. doi: 10.1186/cc8899.

76. Ware LB, Conner ER, Matthay MA. von Willebrand factor antigen is an independent marker of poor outcome in patients with early acute lung injury. Crit Care Med 2001;29(12):2325-2331.

77. Ware LB, Eisner MD, Thompson BT, Parsons PE, Matthay MA. Significance of von Willebrand factor in septic and nonseptic patients with acute lung injury. Am J Respir Crit Care Med 2004;170(7):766-772.

78. Sapru A, Curley MA, Brady S, Matthay MA, Flori H. Elevated PAl-1 is associated with poor clinical outcomes in pediatric patients with acute lung injury. Intensive Care Med 2010;36(1):157-163. doi: 10.1007/s00134-009-1690-2.

79. Arcaroli J, Sankoff J, Liu N, Allison DB, Maloney J, Abraham E. Association between urokinase haplotypes and outcome from infection-associated acute lung injury. Intensive Care Med 2008;34(2):300-307.

80. Prabhakaran P, Ware LB, White KE, Cross MT, Matthay MA, Olman MA. Elevated levels of plasminogen activator inhibitor-1 in pulmonary edema fluid are associated with mortality in acute lung injury. Am J Physiol Lung Cell Mol Physiol 2003;285(1):L20-28.

81. Ware LB, Matthay MA, Parsons PE, Thompson BT, Januzzi $\mathrm{JL}$, Eisner MD; National Heart, Lung, and Blood Institute Acute Respiratory Distress Syndrome Clinical Trials Network. Pathogenetic and prognostic significance of altered coagulation and fibrinolysis in acute lung injurylacute respiratory distress syndrome. Crit Care Med 2007;35(8):1821-1828.

\section{$\triangle$ Correspondencia:}

Dr. Raúl Barrera Rodríguez

Departamento de Bioquímica y Medicina Ambiental Instituto Nacional de Enfermedades Respiratorias Ismael Cosío Villegas. Calzada de Tlalpan Núm. 4502, Colonia Sección XVI, 14080, México, D.F.

Tel: 52-5554871771

Correo electrónico: barrerrarr@hotmail.com raul_barrera@iner.gob.mx

Los autores declaran no tener conflicto de intereses. 